\title{
Cognitive Changes after Cellular Therapy in a Case of Intellectual Disability
}

Keywords: Intellectual disability; Stem cell therapy; Autologous; Bone marrow mononuclear cells; PET CT scan brain

\begin{abstract}
Intellectual Disability (ID) is a developmental disability characterized by sub average intellectual functioning, occurring in $2-3 \%$ of the population. The underlying cause in ID is the sub-functioning of the neurons which significantly affects cognition, learning and adaptive behavior of an individual. The conventional management strategies address specific symptoms observed in ID and not the core pathology. In this case study, we evaluated the symptomatic and functional improvements after intrathecal transplantation of autologous Bone Marrow Mononuclear Cells (BMMNCs) in a 13-yearold girl diagnosed with ID. As a part of the protocol, she was also put on a multidisciplinary rehabilitation program. She underwent two cellular therapies twice at an interval of 11 months. Follow up was done at regular intervals of four and eleven months after each cellular therapy. No major adverse events were recorded post intervention. Improvements in command following, eye contact, sitting tolerance, balance, aggressive behaviour, hyperactivity and clarity of speech were observed over a period of 30 months of first intervention. Functional Independence Score (FIM) score increased from 76 to 78. On comparison of pre-intervention and post intervention after 11 months PET CT scan, improvement in metabolic activity of frontal lobe, cerebellum and the mesial temporal structures was recorded. These objective changes suggest that cellular therapy in combination with rehabilitation improves neuronal functioning, hence improving processing of information in ID. However, larger randomized control studies will be required to demonstrate long term benefits of cellular transplantation in ID.
\end{abstract}

\section{Introduction}

Intellectual Disability (ID) is a developmental disability, characterized by a global deficiency in cognitive abilities and adaptive skills [1]. In practical terms, ID is defined by an Intelligence Quotient (IQ) below 70 , occurring in $2-3 \%$ of the population. ID is highly heterogeneous with respect to the severity of cognitive deficiency and the causes which include both environmental and genetic factors [2]. Assessment of behavioural disorder or psychopathology in people with ID can be problematic due to cognitive or communicative deficits [3]. Investigating the causes of ID and underlying cellular mechanisms are essential to understand the structural and functional basis of ID.

The damage caused to neural and dendritic connectivity in ID can be addressed by a neurorestorative therapy such as cellular therapy. Stem cells have the unique ability of self- renewal, immunomodulation and paracrine regulation through which the underlying brain damage can be repaired $[4,5]$. Hence cellular therapy can be considered as one of the therapeutic modality for ID. In our case study, we evaluated the symptomatic and functional improvements after intrathecal transplantation of autologous Bone Marrow Mononuclear Cells (BMMNCs) in a 13-year-old girl diagnosed with ID with behavioural issues.

\section{Journal of}

\section{Transplantation \& Stem Cell Biology}

\author{
Alok Sharma', Nandini Gokulchandran'1, \\ Hemangi Sane ${ }^{2}$, Suhasini Pai ${ }^{*}$, Pooja \\ Kulkarni ${ }^{2}$, Vaishali Ganwir ${ }^{3}$ and Prerna \\ Badhe $^{4}$
}

${ }^{\prime}$ Department of Medical Services and Clinical research, NeuroGen Brain \& Spine Institute, India

'Department of Research and Development, NeuroGen Brain \& Spine Institute, India

${ }^{3}$ Department of Neurorehabilitation, NeuroGen Brain \& Spine Institute, India

${ }^{4}$ Department of Regenerative Laboratory Services, NeuroGen Brain \& Spine Institute, India

Address for Correspondence

Suhasini Pai, NeuroGen Brain \& Spine Institute, Stem Asia Hospital and Research Centre, Sector-40, Plot No. 19, Palm Beach Road, Seawoods (W) New Mumbai, 400706, India, Tel: 91-9920200400 (or) +9122-25283706 E-mail: publications@neurogen.in

Submission: 01 March, 2017

Accepted: 29 March, 2017

Published: 03 April, 2017

Copyright: (๑) 2016 Sharma A. This is an open access article distributed under the Creative Commons Attribution License, which permits unrestricted use, distribution, and reproduction in any medium, provided the original work is properly cited.

\section{Case Representation}

A 13-year-old female child was born full term normal delivery with a birth weight of $2.5 \mathrm{~kg}$ and cried immediately after birth. During the $5^{\text {th }}$ month of pregnancy, the mother suffered from malaria for which she was administered medications and was hospitalised for 4 days. There was no history of neonatal complications. At the age of 2 years, her parents noticed a delay in speech and cognition for which she was started on medication but no improvements were observed. Gradually she started displaying behavioural issues and hyperactivity. On consultation, she was diagnosed with moderate intellectual disability with behavioural issues. At the age of 5 years, parents consulted an ENT specialist; vision and BERA test were normal. She has been regularly attending special school. IQ test performed at the age of 8 years revealed a score of 42 which suggested moderate intellectual disability.

On detailed assessment prior to the first cellular therapy, she had poor cognition, problem solving abilities, memory and visual perceptual skills. Following commands was difficult with behavioural issues like throwing temper tantrums, increased stubbornness, crying without reason. Speech was not clear and was repetitive. She had difficulty in adjusting to new places and new people. She could not read or write. Attention span was poor and hence she could not attend school for full time. Maximum assistance was required in eating, grooming, bathing and dressing while minimal assistance in toileting. She had modified independence in bladder management, transfer and ambulation. PET CT scan brain revealed reduced Fludeoxyglucose (FDG) uptake in frontal cortex, cerebellum, amygdala, hippocampus and parahippocampal gyrus. Electroencephalogram (EEG) was 
Citation: Sharma A, Gokulchandran N, Sane H, Pai S, Kulkarni P, et al. Cognitive Changes after Cellular Therapy in a Case of Intellectual Disability. J Transplant Stem Cel Biol. 2017;4(1): 4.

normal in sleep state with no potential epileptiform activity observed. MRI with DTI suggested no intracranial abnormality. She scored 76 on Functional Independence Measure (FIM).

\section{Material and Methods}

The selection of the patient was based on the World Medical Associations Helsinki declaration [6]. The protocol was reviewed and ethical approval was taken from Institutional Committee for Stem Cell Research and Therapy (IC-SCRT). The procedure of cellular therapy was explained in detail and a duly filled informed consent was obtained from the parents prior to the therapy.

Granulocyte-Colony Stimulating Factor (G-CSF) (300 mcg) injections were administered subcutaneously, 72 hours and 24 hours prior to bone marrow aspiration. On the day of transplantation, 100 $\mathrm{ml}$ bone marrow was aspirated from the left anterior superior iliac spine under local anesthesia, using bone marrow aspiration needle and was collected in heparinized tubes. The BMMNCs were separated from the aspirate using density gradient method. Manually, the cell viability was calculated using Trypan Blue dye which was confirmed by TALI machine using propidium iodide. Fluorescence Activated Cell sorting (FACS) analysis showed CD34+ count to be $0.86 \%$. The separated cells were diluted with cerebrospinal fluid (CSF) as it has factors that are known to promote cell growth [7].

The cells were injected intrathecally at the level between L4 and L5. Simultaneous intravenous administration of $1 \mathrm{gm}$ methyl prednisolone in $500 \mathrm{ml}$ of Ringer Lactate solution was carried out to decrease immediate inflammation and to enhance the survival of the injected cells. Total numbers of cells injected were 3x108 with $98 \%$ viability.

As a part of the protocol, the patient was given intensive neurorehabilitation therapy followed by cellular therapy. It included a multidisciplinary rehabilitation protocol comprising of psychological intervention, speech therapy and occupational therapy. Psychological intervention aimed at cognitive development and improving social interaction. Speech therapists helped with pronunciation of words, sound production, pitch control and improving communication skills. The occupational therapy interventions included both restorative and adaptive strategies to improve performance of the Activities of Daily Living (ADLs).

The patient was discharged at one-week post transplantation and was advised to continue the rehabilitation at home. The follow up assessment was conducted at four and eleven months after the intervention. PET CT scan brain was repeated to determine metabolic changes in the brain 11 months after first cellular therapy. In view of the improvements observed after the treatment, the patient underwent second cellular transplantation 11 months after the first transplantation. The transplantation procedure was replicated. Total numbers of cells injected were $96 \times 10^{6}$ with $96 \%$ viability. The patient was again followed up at four and eleven months after second cellular therapy.

\section{Results}

Within four months of $1^{\text {st }}$ cellular therapy, hyperactivity had reduced along with improvement sitting tolerance and attention span. Behavioral issues like throwing things out, laughing and crying without reason and temper tantrums reduced. She could dress herself with initial assistance from mother. Speech clarity had improved. There was minimal improvement in eye contact.

Eleven months after the $1^{\text {st }}$ cellular therapy, hyperactivity reduced further. Reaction time while following commands reduced as she would respond within 2-3 repeated instructions. Comprehension and understanding improved significantly. She started doing household activities along with her mother. Transitions were easier with improved balance. All the other improvements were maintained in these months. PET CT scan brain recorded accentuated FDG uptake in the bilateral mesial temporal structures, frontal lobe, thalamus and cerebellum FIM score increased from 76 to 78 (Figure 1).

In view of the improvements, the patient underwent $2^{\text {nd }}$ cellular therapy. Thereafter, the patient was followed up after 4 months (15 months after the $1^{\text {st }}$ cellular therapy). Attention span and concentration increased to 30 minutes with proper eye contact. She would now try to initiate conversation with others. Memory improved as she could recollect all the happenings in school and report it to the mother. Increase in hyperactivity and aggressive behavior was observed a few instances.

After 11 months of $2^{\text {nd }}$ cellular therapy, awareness regarding people and the surrounding was better than before. Multitasking was possible with proper judgment. Clarity of speech improved further. All the other improvements were maintained. Hyperactivity was confined to places which were unknown to her.

\section{Discussion}

Intellectual Disability (ID) is characterized by sub average intellectual functioning with concurrent deficits in communication, self-care, home living, social skills etc [1]. ID is associated with abnormalities in dendritic branching and connectivity of the neuronal network which severely limits its ability to process information [8-10]. Mild forms of ID have been linked to diminutive changes in brain microanatomy, including relevant areas of the brain like
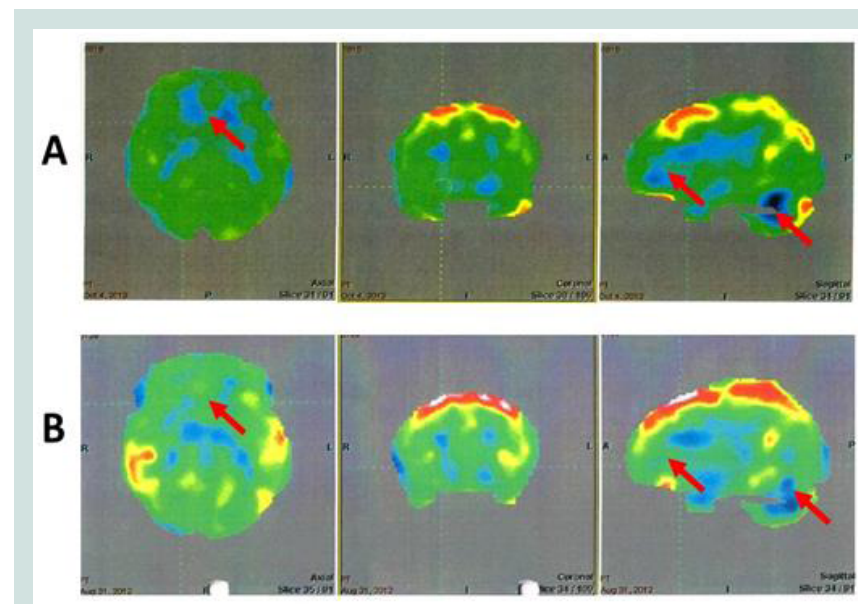

Figure 1: The top row $(A)$ indicates the 18F-FDG image before cellular therapy and below row (B) indicates the 18F-FDG image after cellular therapy showing increased metabolic uptake in the frontal cortex, thalamus and cerebellum. 
Citation: Sharma A, Gokulchandran N, Sane H, Pai S, Kulkarni P, et al. Cognitive Changes after Cellular Therapy in a Case of Intellectual Disability. J Transplant Stem Cel Biol. 2017;4(1): 4

cerebral cortex and hippocampus [11]. Defects in synaptogenesis, synaptic activities and plasticity are crucial cellular processes that are responsible for cognitive impairment, especially in postnatal stage during which learning and acquisition of intellectual abilities and emotional behaviour occurs [12]. With a growing prevalence of ID, it is vital to explore new avenues of diagnosis and management for ID. Due to unknown pathology and lack of reliable cognitive assessment tools, it is difficult to establish a definitive treatment modality. The conventional treatment strategies such as psychological and behavioural intervention, occupational therapy and speech therapy aim towards alleviating specific symptoms observed in ID [13]. However, these strategies do not address the core neuropathology. Recently cellular therapy has gained attention as a potential therapeutic strategy in various neurological disorders [4,5]. To study its effect in ID, we administered autologous BMMNC to the patient, intrathecally.

The safety of autologous Bone Marrow Mononuclear Cells (BMMNCs) has been well established in previous clinical studies as they are devoid of immune rejection and do not exhibit any adverse effect [14]. Studies have shown that bone marrow derived cells can differentiate into mature neurons or glial cells $[15,16]$. In preclinical studies, these cells have improved the underlying neurological deficit by regenerating into neural cells $[17,18]$. BMMNCs have a unique property of homing into the damaged areas and engrafting into the injured cells and preventing the endogenous cells from damage [19]. The repair process occurs through paracrine mechanisms by secretion of certain factors which are postulated to promote angiogenesis, neurogenesis, immunomodulation and reduce inflammatory processes $[20,21]$. These findings increase the prospects of BMMNCs to be considered therapeutically beneficial for patients with neurological conditions.

The patient showed a significant change in her daily activities and improvement in quality of life. Improvements in memory, cognition, learning ability, behaviour were also evident. Positron Emission Tomography (PET) with 2-[fluorine-18] Fluoro-2-deoxy-D-glucose (FDG) is useful imaging modality for the diagnosis of neurological disorders. FDG PET for the evaluation of cognitive impairment has been found to help detect the earliest changes in neurodegenerative disorders [22]. PET CT scan brain was performed 11 months after the $1^{\text {st }}$ intervention to study the effect of cellular therapy on the metabolic activity of the brain. The metabolic changes in the brain recorded in PET CT post cellular therapy corresponded to the functional improvements. The improved social participation and command following in the patient can be attributed to improved frontal lobe functioning [23]. Enhanced memory, learning ability, cognitive skills, emotional learning and decision making correlated with increased FDG uptake in mesial temporal structures [24]. The increase in FDG uptake in the cerebellum is responsible for improvements in balance, coordination and fine motor activities as reflected in the PET scan in the patient [25].

The clinical improvements observed in the subject can be attributed to the cumulative effect of two cell transplantations and multidisciplinary neurorehabilitation, supporting the observation that a combination of cellular therapy and exercise training results in significant functional improvement through neuro-facilitation [26].
There were no adverse effects observed in the patient post cellular therapy.

\section{Limitations}

The reported case is a single case report and there was no control available to study the independent effect of rehabilitation or cellular therapy. The improvements were noticed after combining cellular therapy to the standard rehabilitation regime. This suggests that cellular therapy has a vital role in improving the neuronal function which was supported by the PET CT brain findings.

\section{Conclusion}

The findings in this case report shows that cellular therapy can be beneficial in treating the neurological deficits in ID. Cellular therapy can be regarded as a novel treatment to improve the overall functioning of the brain in ID and to improve the quality of life of patients with ID. PET CT scan can be used an objective scale to determine the structural changes occurring in the brain during the course. However, larger randomized control studies will be required to demonstrate long term benefits of cellular transplantation in ID.

\section{References}

1. Baroff GS, Olley JG (1999) Mental retardation: nature, cause and management, $\left(3^{\text {rd }}\right.$ edn). Brunner/Mazel-Taylor and Fransis Group, Philadelphia, PA, USA.

2. Chiurazzi P, Pirozzi $F$ (2016) Advances in understanding - genetic basis of intellectual disability. F1000Res 5. pii: F1000 Faculty Rev-599.

3. Smith KR, Matson JL (2010) Behavior problems: differences among intellectually disabled adults with co-morbid autism spectrum disorders and epilepsy. Res Dev Disabil 31: 1062-1069.

4. Sharma A, Gokulchandran N, Chopra G, Kulkarni P, Lohia M, et al. (2012) Administration of autologous bone marrow-derived mononuclear cells in children with incurable neurological disorders and injury is safe and improves their quality of life. Cell transplant 21 Suppl 1: S79-S90.

5. Sharma A, Gokulchandran N, Shetty A, Sane H, Kulkarni P, et al. (2013) Autologous bone marrow mononuclear cells may be explored as a novel potential therapeutic option for autism. J Clin Case Rep 3: 1-4.

6. Carlson RV, Boyd KM, Webb DJ (2004) The revision of the Declaration of Helsinki: past, present and future. Br J Clin Pharmacol 57: 695-713.

7. Hagberg B, Kyllerman M (1983) Epidemiology of mental retardation--a Swedish survey. Brain Dev 5: 441-449.

8. Huttenlocher PR (1974) Dendritic development in neocortex of children with mental defect and infantile spasms. Neurology 24: 203-210.

9. Levenga J, Willemsen $\mathrm{R}(2012)$ Perturbation of dendritic protrusions in intellectual disability. In: Dierssen M, de la Torre FR (Eds). Down syndrome: from understanding the neurobiology to therapy. 197: 153

10. KaufmannWE, Moser HW (2000) Dendritic anomalies in disorders associated with mental retardation. Cerebral Cortex 10: 981-991.

11. Whishaw IQ, Jarrard LE (1996) Evidence for extrahippocampal involvement in place learning and hippocampal involvement in path integration. Hippocampus 6: 513-524.

12. Chelly J, Khelfaoui M, Francis F, Chérif B, Bienvenu T (2006) Genetics and pathophysiology of mental retardation. Eur J Hum Genet 14: 701-713.

13. Kottorp A, Hällgren M, Bernspang B, Fisher AG (2003) Client-centred occupational therapy for persons with mental retardation: Implementation of an intervention programme in activities of daily living tasks. Scand J Occup Ther 10: $51-60$.

14. Geffner LF, Santacruz P, Izurieta M, Flor L, Maldonado B, et al. (2008) 
Citation: Sharma A, Gokulchandran N, Sane H, Pai S, Kulkarni P, et al. Cognitive Changes after Cellular Therapy in a Case of Intellectual Disability. J Transplant Stem Cel Biol. 2017;4(1): 4

ISSN: $2374-9326$

Administration of autologous bone marrow stem cells into spinal cord injury patients via multiple routes is safe and improves their quality of life: comprehensive case studies. Cell Transplant 17: 1277-1293.

15. Muñoz Elías G, Woodbury D, Black IB (2003) Marrow stromal cells, mitosis, and neuronal differentiation: stem cell and precursor functions. Stem Cells 21: 437-448.

16. Sanchez-Ramos J, Song S, Cardozo-Pelaez F, Hazzi C, Stedeford T, et al (2000) Adult bone marrow stromal cells differentiate into neural cells in vitro. Exp Neurol 164: 247-256.

17. Chopp M, Zhang XH, Li Y, Wang L, Chen J, et al. (2000) Spinal cord injury in rat: treatment with bone marrow stromal cell transplantation. Neuroreport 11: $3001-3005$

18. Akiyama Y, Radtke C, Kocsis JD (2002) Remyelination of the rat spinal cord by transplantation of identified bone marrow stromal cells. J Neurosci 22: 6623-6630.

19. Yagi H, Soto-Gutierrez A, Parekkadan B, Kitagawa Y, Tompkins RG, et al (2010) Mesenchymal stem cells: mechanisms of immunomodulation and homing. Cell Transplant 19: 667-679.
20. Payne N, Siatskas C, Barnard A, Bernard C (2011) The prospect of stem cells as multi-faceted purveyors of immune modulation, repair and regeneration in multiple sclerosis. Curr Stem Cell Res Ther 6: 50-62.

21. Chen PM, Yen ML, Liu KJ, Sytwu HK, Yen BL (2011) Immunomodulatory properties of human adult and fetal multipotent mesenchymal stem cells. $J$ Biomed Sci 18: 49 .

22. Del Sole A, Clerici F, Chiti A, Lecchi M, Mariani C, et al. (2008) Individual cerebral metabolic deficits in Alzheimer's disease and amnestic mild cognitive impairment: an FDG PET study. Eur J Nucl Med Mol Imaging 35: 1357-1366.

23. Whishaw IQ, Jarrard LE (1996) Evidence for extrahippocampal involvement in place learning and hippocampal involvement in path integration. Hippocampus 6: 513-524.

24. Sarter M, Markowitsch HJ (1985) Involvement of the amygdala in learning and memory: a critical review, with emphasis on anatomical relations. Behav Neurosci 99: 342-380.

25. Houk JC, Miller LE (2001) Cerebellum: Movement regulation and cognitive functions. eLS: $1-6$.

26. Fabel K, Wolf S, Ehninger D, Babu H, Galicia PL, et al. (2009) Additive effects of physical exercise and environmental enrichment on adult hippocampal neurogenesis in mice. Front Neurosci 3: 50. 